\title{
Enhancing the transdermal penetration of nanoconstructs: could hyaluronic acid be the key?
}

\author{
"For further applications of nanoconstructs in dermatology, we need efficient \\ transdermal delivery carriers of the nanoconstructs."
}

\begin{abstract}
Keywords: hyaluronic acid $\bullet$ nanoconstructs $\bullet$ nanomedicines $\bullet$ transdermal delivery
\end{abstract}
Nanoconstructs, such as liposomes, polymeric micelles, gold nanoparticles, carbon nanomaterials and nanocrystalline quantum dots, have been widely investigated for diagnostic, bioimaging and therapeutic applications [1]. Nanoconstructs have been also extensively explored in the field of dermatology. For example, liposomes and polymeric nanoparticles loaded with drugs were applied as topical administration agents for the treatment of skin diseases such as psoriasis, dermatitis and skin cancer $[2,3]$. Titanium dioxide and zinc oxide $(\mathrm{ZnO})$ nanoparticles have been used as sun-screen formulation for the protection of skin from UV by the scattering, absorption and reflection of UV [4]. Silver nanoparticles are commercially used as wound and burn dressing agents with antibacterial effects [5]. Quantum dots and $\mathrm{ZnO}$ nanoparticles were utilized as bioimaging agents for the diagnosis of skin diseases [6,7]. Moreover, gold nanoconstructs and carbon nanomaterials have been actively investigated as promising agents for the photothermal ablation therapy of skin cancers due to their high light-to-heat conversion capability $[8,9]$.

For further applications of nanoconstructs in dermatology, we need efficient transdermal delivery carriers of the nanoconstructs. The transdermal delivery has several advantages over other administration routes, such as oral delivery and needle based injection. The advantages include noninvasive treatment, self-administration, improved patient compliance and avoidance of hepatic first-pass metabolism or digestion system [10]. Despite these benefits, the low skin permeability of nanoconstructs such as polymers, proteins, hydrophilic drugs and nanoparticles limited their wide applications to the transdermal delivery. To facilitate the transdermal delivery of nanoconstructs, additional treatments have been adopted using penetration enhancers, iontophoresis, ultrasound and microneedles [11]. However, these methods require physical perturbations to the skin tissue, causing skin damage in some cases [12]. A noninvasive molecular carrier for transdermal delivery would have compelling advantages. The understanding for the characteristics of skin layers can be a good starting point for the development of transdermal delivery carriers of nanoconstructs.

Stratum corneum (SC), the outermost skin layer, is the main barrier composed of densely packed dead cells forming hydrophobic surfaces. For the penetration of hydrophobic SC, hydrophobic molecules have clear benefits over hydrophilic molecules. The hydrophobic molecules can infiltrate into densely packed lipid layers in SC. However, nanoconstructs are usually formulated to have hydrophilic surfaces enhancing the physiological stability in the biological conditions. This contradicting requirement of hydrophobicity and hydrophilicity for transdermal delivery should be reconciled to enhance the physiological stability and the skin permeability by lipid disruption in SC.

Recently, hyaluronic acid (HA) has been investigated as a promising transdermal delivery carrier. HA is a naturally occurring linear polysaccharide composed of repeating units of D-glucuronic acid and $N$-acetyl-

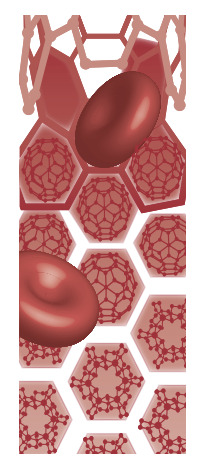

Ho Sang Jung

Department of Materials Science \& Engineering, Pohang University of Science \& Technology (POSTECH), San 31, Hyoja-dong, Nam-gu, Pohang, Kyungbuk 790-784, Korea

\section{Ki Su Kim}

Wellman Center for Photomedicine, Department of Dermatology, Harvard Medical School \& Massachusetts General Hospital, 65 Landsdowne St UP-5, Cambridge, MA 02139, USA

\section{Seok Hyun Yun}

Wellman Center for Photomedicine, Department of Dermatology, Harvard Medical School \& Massachusetts General Hospital, 65 Landsdowne St UP-5, Cambridge, MA 02139, USA

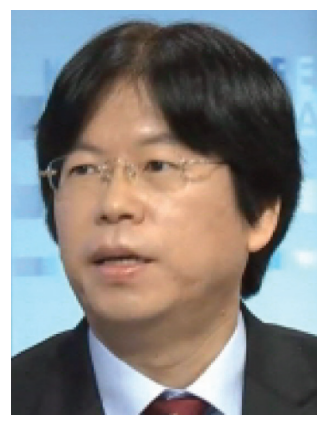

Sei Kwang Hahn

Author for correspondence: Department of Materials Science \& Engineering, Pohang University of Science \& Technology (POSTECH), San 31, Hyoja-dong, Nam-gu, Pohang, Kyungbuk 790-784, Korea

Tel.: +82 542792159 skhanb@postech.ac.kr 
D-glucosamine. HA is abundant in the epithelial, connective and neural tissues. HA is biocompatible, biodegradable, nonimmunogenic and nontoxic. HA has been regarded as one of the best biopolymers for biomedical applications including drug delivery and tissue engineering. HA is one of the major components in the skin and plays an important role in extracellular matrix. HA also participates in tissue hydrodynamics, cell movement and proliferation, and interactions with a variety of cells with HA receptors like CD44 and RHAMM. Furthermore, HA is viscoelastic and hygroscopic enabling cosmetic and cosmeceutical applications. Brown et al. reported that radiolabeled HA with a high molecular weight of $360-400 \mathrm{kDa}$ could pass through SC, epidermis and dermis layers of mouse and human skins [13]. Interestingly, highmolecular-weight HA was reported to inhibit the malignant tumor progression [14]. The antitumor effect of HA can contribute to the treatment of skin cancers using HA conjugated with various anticancer drugs.

\section{"...hyaluronic acid can be used as a promising transdermal delivery carrier of nanoconstructs for diagnostic and therapeutic applications."}

Although the exact mechanism for the transdermal delivery of HA derivatives is not fully proven yet, there are possible reasons and evidences for the transdermal delivery of HA derivatives across the skin barrier. First, HA is a hygroscopic polyanion that keeps high water content within its structural backbone. The skin hydration is important for the enhancement of skin permeability. When SC is exposed to water, the tissue swells and forms paths for the molecular transport into the skin. HA derivatives can hydrate SC, opening penetration routes and facilitating transdermal delivery of tethered cargos. Along with biocompatibility, viscoelasticity and antiwrinkle effect, the ability to hydrate the skin has made $\mathrm{HA}$ as a unique molecule for various cosmetic and cosmeceutical applications.

Second, HA is an amphiphilic liner polysaccharide at the molecular level. HA is well known as one of the hydrophilic polysaccharides, but structural analysis has revealed at the molecular level that there is a hydrophobic patch domain consisting of eight $\mathrm{CH}$ groups [15]. In addition, NMR and rotary-shadowing electron microscopy showed that hydrophobic patch domain of HA could make a complex with phospholipids [16,17]. The structural hydrophobic patch domain of HA may interact with lipid components in SC during penetration and disrupt skin barriers, enhancing the skin permeability of HA derivatives. Similarly, gold nanoparticle was reported to disrupt
SC by the interaction of lipid-gold nanoparticle [18] We previously compared the transdermal delivery of HA conjugated nanographene oxide (NGO) and polyethylene glycol conjugated NGO in normal and cancer model mice [9]. While the nonfouling polyethylene glycol inhibited the skin penetration of NGO in both normal and cancer model mice, HA conjugated NGO with a dimension of $200 \mathrm{~nm}$ appeared to be transdermally delivered through the cancerous skin.

Third, HA receptors on skin resident cells facilitate the transdermal delivery of HA derivatives. HA receptors are highly expressed in the keratinocytes in epidermis and fibroblasts in dermis. We previously visualized the transdermal delivery of HA-human growth hormone (hGH) conjugate in Balb/c mice [11]. After topical delivery of HA derivatives, the HA receptor mediated internalization into skin cells appeared to facilitate the transdermal delivery of HA derivatives, forming the concentration gradient from the top to the bottom of skin tissues. The bioavailability of hGH in the HA-hGH conjugate after transdermal delivery was as high as $15 \%$, reflecting the feasibility of $\mathrm{HA}$ as a promising transdermal delivery carrier of protein drugs. The transdermal delivery of HA derivatives was more efficient in cancerous skin. On the surface of skin cancer cells, especially melanoma, highly expressed HA receptors can internalize HA derivatives via the HA receptor mediated endocytosis. The effective transdermal delivery of HA-NGO conjugates for the photothermal ablation therapy of skin cancer might be also related with HA receptors locally overexpressed around cancerous skin tissues. The transdermally delivered HA-NGO accumulated specifically in leaky and damaged skin cancer regions, and the near-infrared laser irradiation successfully ablated the tumor tissues [9].

In summary, with the development of various nanoconstructs, their transdermal delivery has been actively investigated for facile biomedical applications. HA could be the key molecule to enhance the transdermal penetration of nanoconstructs by the synergetic effect of the following factors. The hygroscopic HA molecules hydrate skin tissues and open the transdermal pathway of conjugated cargos. The structural hydrophobic patch domains in HA molecule help the penetration of HA and its cargo disrupting lipid structure of SC. HA receptors expressed on skin cells and highly expressed HA receptors on cancerous skin cells can facilitate the continuous penetration of HA derivatives via the HA receptor mediated internalization into the cells. Taken together, HA can be used as a promising transdermal delivery carrier of nanoconstructs for diagnostic and therapeutic applications. 
Financial \& competing interests disclosure

This work was financially supported by the Converging Research Center Program through the National Research Foundation of Korea (NRF) funded by the Ministry of Education, Science and Technology (2009-0081871). This study was also supported by Mid-career Researcher Program through NRF grant funded by the MEST (No. 2012R1A2A2A06045773).
The authors have no other relevant affiliations or financial involvement with any organization or entity with a financial interest in or financial conflict with the subject matter or materials discussed in the manuscript apart from those disclosed.

No writing assistance was utilized in the production of this manuscript.

\section{References}

1 Kim BYS, Rutka JT, Chan WCW. Nanomedicine. N. Engl. J. Med. 363, 2434-2443 (2010).

2 Schafer-Korting M, Mehnert W, Korting HC. Lipid nanoparticles for improved topical application of drugs for skin diseases. Adv. Drug Deliv. Rev. 59, 427-443 (2007).

3 Zhang Z, Tsai PC, Ramezanli T et al. Polymeric nanoparticles-based topical delivery systems for the treatment of dermatological diseases. Wiley Interdiscip. Rev. Nanomed. Nanobiotechnol. 5, 205-218 (2013).

4 Nel A, Xia T, Madler L et al. Toxic potential of materials at the nanolevel. Science 311, 622-627 (2006).

5 Kumar A, vemula PK, Ajayan PM et al. Silver-nanoparticleembedded antimicrobial paints based on vegetable oil. Nat. Mater. 7, 236-241 (2008).

6 Larson DR, Zipfel WR, Williams RM et al. Water-soluble quantum dots for multiphoton fluorescence imaging in vivo. Science 300, 1434-1436 (2003).

7 Kuo TR, Wu CL, Hsu CT et al. Chemical enhancer induced changes in the mechanisms of transdermal delivery of zinc oxide nanoparticles. Biomaterials 30, 3002-3008 (2009).

8 Lu W, Xiong C, Zhang G et al. Targeted photothermal ablation of murine melanomas with melanocyte-stimulating hormon analog-conjugated hollow gold nanospheres. Clin. Cancer Res. 15, 876-886 (2009).

9 Jung HS, Kong WH, Sung DK et al. Nanographene oxidehyaluronic acid conjugate for photothermal ablation therapy of skin cancer. ACS Nano 8, 260-268 (2014).
10 Prausnitz MR, Langer R. Transdermal drug delivery. Nat. Biotechnol. 26, 1261-1268 (2008).

11 Yang JA, Kim ES, Kwon JH et al. Transdermal delivery of hyaluronic acid - human growth hormone conjugate. Biomaterials 33, 5947-5954 (2012).

12 Prausnitz MR, Mitragotri S, Langer R. Current status and future potential of transdermal drug delivery. Nat. Rev. Drug. Discov. 3, 115-124 (2004).

13 Brown TJ, Alcom D, Fraser JR. Absorption of hyaluronan applied to the surface of intact skin. J. Invest. Dermatol. 113, 740-746 (1999).

14 Tian X, Azpurua J, Hine C et al. High-molecular-mass hyaluronan mediates the cancer resistance of the naked mole rat. Nature 499, 346-349 (2013).

15 Brown MB, Jones SA. Hyaluronic acid: a unique topical vehicle for the localized delivery of drugs to the skin. J. Eur. Acad. Dermatol. Venerol. 19, 308-318 (2005).

16 Ghosh P, Hutadilok N, Adam N et al. Interactions of hyaluronan (hyaluronic acid) with phospholipids as determined by gel permeation chromatography, multi-angle laser-light-scattering photometry and ${ }^{1} \mathrm{H}-\mathrm{NMR}$ spectroscopy. Int. J. Biol. Macromol. 16, 237-244 (1994).

17 Pasquali-Ronchetti I, Quaglino D, Mori G et al. Hyaluronan-phospholipid interactions. J. Struct. Biol. 120, 1-10 (1997).

18 Huang Y, Yu F, Park YS et al. Co-administration of protein drugs with gold nanoparticles to enable percutaneous delivery. Biomaterials 31, 9086-9091 (2010). 